DOI: https://doi.org/10.29105/gmjmx18.35-5

Artículos

\title{
TIPOS DE ARGUMENTACIÓN MORAL Y TEMAS PROTOTÍPICOS DE DISCUSIÓN EN FACEBOOK: UN ESTUDIO CUALITATIVO
}

\section{MORAL ARGUMENTATION AND PROTOTYPICAL THEMES OF DISCUSSION IN FACEBOOK: A QUALITATIVE STUDY}

\section{Rolando Pérez Sánchez}

Universidad de Costa Rica, Costa Rica

(iD) https://orcid.org/0000-0001-6321-2543

\section{Mario Solís Umaña}

Universidad de Costa Rica, Costa Rica

(iD https://orcid.org/0000-0002-9387-342X

\section{Fiorella Jara Sanabria}

Universidad de Costa Rica, Costa Rica

(iD) https://orcid.org/0000-0001-9412-5469

\section{Andrea García Román}

Universidad de Costa Rica, Costa Rica

(iD https://orcid.org/0000-0002-7241-1904

Autor para correspondencia: Rolando Pérez Sánchez, email: rolarez@ gmail.com

\section{Resumen}

Este estudio persigue identificar temas centrales de debate en Facebook, desde la perspectiva de personas usuarias y concerniente a los criterios utilizados para la evaluación moral de dichos temas. Se establece una relación entre los mecanismos socio-cognitivos de la evaluación moral y cinco tipos filosóficos generales de juicio moral. El estudio de dicha correspondencia marca un campo de trabajo poco abordado por la investigación asociada. Se recurrió un diseño cualitativo fenomenológico, y se emplearon entrevistas grupales a un total de 32 estudiantes universitarios costarricenses (43\% mujeres entre 18 y 24 años) para la recolección de información. Los resultados permitieron distinguir cuatro temas centrales a partir de los cuales se define el debate en esta red social. En estos intercambios operan esquemas cognitivos rígidos, cuyo contenido predominante corresponde a dos de los cinco tipos de juicio moral con los que se trabajó, es decir, el teologismo y el deontologismo. Se discuten los alcances, limitaciones e implicaciones del estudio para la comprensión de los intercambios comunicativos en las redes sociales y la formación de una ciudadanía digital.

Palabras clave: cognición, interacción social, psicología de la comunicación, filosofía moral, redes sociales, Internet. 


\begin{abstract}
This study aims to identify central issues of debate on Facebook, as well as the criteria for the moral evaluation of these issues. It looks at the links between the socio-cognitive mechanisms of moral evaluation and five philosophical types moral reasoning. This approach has not been directly addressed in the associated research. The study applies a qualitative phenomenological method. Group interviews were carried out for the data collection with a total of 32 Costa Rican university students (18-24 years old, 43\% women). The results yielded four central issues around which the debate takes place within this social network. Within the lines of Facebook exchanges, there is a rigid cognitive scheme whose content relates to two of the five philosophical types of moral reasoning, namely, theologism and deontologism. The study alludes to the scope, limitations, and implications for the understanding of communicative exchanges in social networks that sheds light on the formation of a digital citizenship.
\end{abstract}

Keywords: cognition, social interactions, communication psychology, moral philosophy, social networks, Internet.

Recibido: 25/08/2021

Aceptado: 14/10/2021

\section{Introducción}

El presente artículo se orienta a estudiar las principales temáticas que se discuten en la red social Facebook, desde la perspectiva de las personas usuarias. Asimismo, se buscó identificar los criterios utilizados en la evaluación moral de dichos temas, a partir de una tipología básica de argumentación moral.

El advenimiento y la masificación de la Internet en la vida cotidiana a nivel global implica el acceso a una nueva fuente de información que ofrece versiones o perspectivas diversas del mundo; incluidas formas de evaluarlo y emitir juicios sobre lo que acontece diariamente. Las características de los intercambios comunicativos en las redes sociales, las temáticas que se discuten y las consecuencias de dichos intercambios en las decisiones de las personas, grupos o instituciones resultan temas cada vez más relevantes en la opinión pública. Dada la relevancia de esta área problemática para la comprensión de la tematización colectiva de problemas sociales, la conformación de la cultura política actual y la formación y mantenimiento de los movimientos sociales, se considera que emprender la investigación empírica sobre el tema resulta_muy pertinente tanto por los aportes científicos como por sus implicaciones sociales.

El debate sobre temas económicos, sociales, políticos o ambientales no acontece sólo en espacios especializados. Los espacios de deliberación se han 
venido diversificando y ampliando de modos inéditos, entre otras cosas, en razón de contextos cotidianos de intercambio intensivo entre ciudadanos, respecto de los cuales las redes sociales ocupan un lugar relevante. Wright et al., (2016) se refieren a estos espacios de construcción de la opinión pública online como "el tercer espacio" a la par del espacio del hogar (primer espacio) y del trabajo (segundo espacio). Se caracterizan por su informalidad, y por niveles disímiles de argumentación en los que acontecen los intercambios (Kotras, 2018).

Facebook se ha convertido, precisamente, en un canal para que personas y grupos realicen sus evaluaciones de lo que sucede en diferentes ámbitos de la sociedad y, de igual manera, produzcan juicios sobre dichos sucesos. Las candidaturas a la presidencia, un proyecto de ley, un suceso violento, actos de corrupción, desastres naturales, pero también el devenir de la vida privada de individuos, son no sólo tematizados, sino también evaluados y juzgados moralmente. Las redes sociales se han consolidado entonces como las principales plataformas para canalizar estas múltiples formas de evaluación de la realidad local y global (Jost et al., 2018). Facebook en particular se mantiene aún como la principal aplicación en donde acontecen estos intercambios, tanto entre jóvenes como entre personas adultas (Pérez Sánchez, 2016; Quirós, 2016).

Para el estudio de los tipos y contenidos de la argumentación moral que permiten a una persona aceptar o refutar determinadas aseveraciones hechas en las redes sociales, esta investigación recurrió tanto a los aportes del estudio de la cognición social sobre la evaluación moral y la toma de decisiones, como a la producción filosófica sobre marcos conceptuales y concepcionales desde los cuales se suele caracterizar el juicio moral. No son comunes las publicaciones locales e internacionales en esta línea de trabajo o que pretendan trabajar de forma conjunta con los recursos generados por ambos campos de estudio, de allí que la investigación se presenta como un aporte para el abordaje interdisciplinario del contenido de los intercambios en redes sociales.

Desde la investigación sociocognitiva se ha planteado la presencia de un modelo dual, que supone un procesamiento automático, orientado por heurísticos, y un modelo controlado, que recurre a toma de decisiones conscientes (Brand, 2016). La evaluación moral se produce usualmente gracias a heurísticos que, como respuesta a una emoción (Sauer, 2017), permiten atribuir causalidad, responsabilidad y asumir una actitud con respecto a la situación o persona evaluada (Sloman et al., 2009). Es posible emitir un juicio moral, que implica una elaboración controlada de la evaluación inicial y permite argumentar la evaluación inicial elaborada de forma automática. Este proceso de evaluación se sucede en el marco de un proceso de elaboración del juicio moral complejo que consta de tres niveles: se inicia a nivel grupal, es decir, el o los grupos de referencia a los cuales pertenecen las personas y que proveen de normas que orientan la evaluación moral. El nivel intermedio es el de la interacción, el espacio donde se debate sobre la evaluación de las acciones, ya sea de conformidad a una norma o a normas rivales. Por último, el nivel individual, en el que se 
toman las decisiones a partir del vínculo con los otros dos niveles (Tarantola, 2017).

Ahora bien, el procesamiento cognitivo asociado a las evaluaciones morales no se lleva a cabo por individuos aislados. Por el contrario, acontece en el marco de procesos comunicativos y del intercambio de argumentos que sirven de insumos y determinan la evaluación moral (Mercier y Sperber, 2017). Precisamente, los intercambios en las redes sociales aportan de forma significativa en la toma de decisiones y el juicio moral acerca de un tema de discusión. La investigación filosófica es fundamental en este contexto, puesto que uno de sus campos distintivos es precisamente el estudio de sistemas normativos morales -sistemas que se comprenden en buena medida a través de la oferta de razones para la acción (morales y no morales). Conviene tratar este aspecto filosófico del estudio con mayor detenimiento. La evaluación moral se entiende básicamente como razonamiento justificatorio y directivo de la acción, en el que se adjudica valor a dicha acción, usualmente valor intrínseco, relativo al bien contenido en la acción, a la virtud que supone la acción o a la adecuación a normas de carácter universal y absoluto (Korsgaard, 2000; Raz, 2013). Estos tres elementos de juicio moral, el bien, la virtud, la norma universal marcan una tipología tripartita con ciertas ventajas para el análisis, no sólo porque son muy recurrentes sino también porque se muestran muy compactas e incluso económicas para el análisis de los datos obtenidos. No obstante, conviene abrir un poco más el espectro tipográfico con el fin de capturar otros elementos que los intercambios expresan. Por esta razón se recurre a cinco tipos de juicio moral (cinco sistemas morales), con los cuales se logra cumplir con los objetivos de la investigación. Por supuesto, estos cinco tipos no agotan los sistemas morales ni son los únicos que pueden rastrearse en la literatura, pero permiten de modo suficiente estructurar las evaluaciones morales en las redes sociales:

1) Consecuencialismo: se trata de un modelo cuya fórmula simple dice: un acto correcto es aquel que trae consigo las mejores consecuencias. La versión más elaborada del consecuencialismo es el utilitarismo, según el cual lo correcto o incorrecto de un acto se dice en función de la maximización del bien (felicidad, placer, bienestar) para el mayor número de individuos receptores del beneficio (Crisp, 1998; Scanlon, 2007).

2) Deontologismo: se trata de un modelo cuya fórmula simple consiste en sostener que un acto correcto es aquel que sigue la norma correcta. La norma correcta, expresada a modo de principio universal de la razón, será el criterio fundacional de la acción moralmente justificada (Kant, 1998 [1785]). El peso de la cuestión recae en la norma y, una vez entendida la norma correcta, quedamos frente al deber de actuar en correspondencia con ella.

3) Virtuosismo: es un modelo cuya fórmula consiste en sostener que un acto "correcto" superviene al agente virtuoso (Slote, 2007). Así, un acto es correcto en tanto acto que "materializa" una virtud, y la virtud es la "manifestación" del agente virtuoso. Los 
vicios, entendidos como actos que se alejan de lo correcto estarían contenidos dentro de este modelo, precisamente evaluando los actos que se alejan de la virtud. El peso del juicio moral está en la "naturaleza" del agente, y la virtud es la expresión de esa naturaleza virtuosa, mientras que el vicio es lo que se aparta de dicha naturaleza.

4) Contractualismo: es un modelo semejante al deontologismo, según el cual un acto correcto es aquel que sigue una norma, pero esta es producida a través del acuerdo entre las partes interactuantes (Rawls, 2005; Scanlon, 2007).

5) Teologismo: Se trata de un modelo altamente "externalista", según el cual un acto correcto es aquel que sigue los designios de otro (agente moral superior tipo Dios, el destino, etc.). En la literatura reciente se suele denominar a este modelo Divine command theory (Teoría del Mandato Divino) y se identifica con el voluntarismo teológico (Quinn, 2007).

El contracturalismo y el teologismo pueden verse como subtipos del deontologismo, al tomar como referencia una norma general o abstracta, pero el sustento de dicha norma permite identificarlos como tipos diferentes. Las éticas no religiosas, señala Parfit (2011), apenas comienzan a ser distinguibles en la teorización ética. Vale entonces tratar de distinguir bien entre teorías que se acercan o se alejan de la teologización del juicio moral.

Desde el punto de vista filosófico-moral, el análisis de los intercambios no significa que se proceda a justificar un posicionamiento moral (lo cual sería caer en la así llamada "falacia naturalista"), sino que de tales intercambios se obtiene una ubicación del razonamiento moral a través de sus tipos. Es una explicación del razonamiento moral, que a su vez, desde el punto de vista de quienes generan los juicios o las aseveraciones, es también una justificación de su posicionamiento; pero conviene no confundir la justificación de la acción o posicionamiento moral (lo dicho en primera persona, por parte de las personas participantes) con lo dicho en este estudio sobre el razonamiento moral (lo dicho en tercera persona, desde el punto de vista explicativo de quienes realizan la investigación).

La investigación previa sobre evaluación moral y redes sociales se concentra en las variables asociadas a la evaluación, no al contenido de la evaluación como tal. Johnen et al. (2018) señalan que los contenidos que evocaron mayor carga emocional, específicamente mayor arousal, eran más cuestionables moralmente que los de la condición de bajo arousal moral. Miller et al. (2018) llevan a cabo un estudio en el que se valoró el efecto del nivel de satisfacción con el mensaje y el tipo de argumentación empleada. Al respecto encontraron que en las personas satisfechas fue frecuente el uso de estrategias argumentativas de ataque $y$ de comparación para establecer el valor de las figuras políticas. Los insatisfechos igualmente emplearon la comparación con figuras políticas pasadas y, además, agregaron argumentos de predicción futura. Finalmente, los argumentos neutrales, utilizaron la precaución como eje argumentativo central, 
asimismo que imprudente y lleno de especulaciones al emitir un juicio sobre la administración política.

Alberici \& Milesi (2016) analizan las características que presentan los intercambios comunicativos al debatir sobre un tema en las redes sociales. El sesgo de confirmación y el sesgo de desconfirmación determinan el debate. Los argumentos neutrales o con dos puntos de vista tienen efectos más débiles en el reforzamiento de actitudes que los argumentos de un solo punto de vista. Las personas con actitudes positivas más fuertes hacia un tema se ven más afectadas por los argumentos confirmatorios o contradictorios que por los argumentos neutrales. Una mayor proporción de los participantes expuestos a argumentos mixtos o neutrales modificaron sus actitudes. Los participantes con actitudes moderadas expuestos a argumentos contradictorios fueron más propensos a cambiar de bando que los que tenían actitudes moderadas, pero estuvieron expuestos a argumentos confirmatorios (Karlsen et al., 2017).

Brady et al. (2017) encuentran que las emociones morales motivan a los individuos a compartir y discutir sus ideas, más que las emociones no morales. Además, encuentran que la expresión de la emoción moral ayuda a la difusión en las redes políticas dentro del grupo más que en redes grupales generales.

El único estudio directo, sobre la relación entre modelos filosóficos de evaluación moral y los intercambios en las redes sociales, encontrado en la literatura internacional es el de Yoon (2011). El autor presenta una propuesta empírica para el estudio del razonamiento moral en la Internet. Según Yoon
(2011), los argumentos éticos con los cuales se procede a emitir un juicio acerca de un fenómeno se sustentan en diferentes concepciones morales. El juicio resultante producto del tipo de razonamiento moral al que se recurra va a definir la expectativa de comportamiento de la persona con respecto al objeto evaluado.

Se plantean como preguntas de investigación la siguientes: ¿cuáles son situaciones prototípicas relevantes de razonamiento moral en la red social Facebook en jóvenes universitarios usuarios de la red social Facebook?, ¿cuáles son los contenidos de la evaluación moral más relevantes?, ¿qué modelos de razonamiento moral son los primordiales?

\section{Método}

\section{Diseño}

Estudio cualitativo transversal de tipo fenomenológico (Cresswell, 2013). Se pretende identificar escenarios prototípicos de evaluación y enjuiciamiento moral que puedan presentarse cotidianamente en Facebook.

\section{Participantes}

32 estudiantes universitarios de diferentes carreras; $43 \%$ mujeres entre los 18 y 24 años. Todas las personas participantes firmaron previamente un consentimiento informado. El estudio fue aprobado por el Comité Ético Científico de la Universidad de Costa Rica. 


\section{Procedimiento de recolección de la información}

Se recurrió a la técnica de entrevista grupal (Flick, 2009). Esta técnica se diferencia de los grupos de discusión o de enfoque, en que posee una moderación más directiva, considerando las perspectivas, evaluaciones o valoraciones de cada una de las personas entrevistadas ocurridas en un contexto de comunicación grupal.

Se llevaron a cabo nueve entrevistas grupales que oscilaron entre dos y seis participantes, con el fin de identificar situaciones cotidianas que demanden un juicio moral en el contexto de la comunicación en Facebook. Se dirigieron a identificar escenarios de tipo político, intergrupal, interpersonal y acciones individuales de situaciones públicas. Para ello se construyó una guía de conducción de los grupos que permitió distinguir dichos escenarios y las formas cómo fueron evaluados en esta red social. Aspectos indagados con la guía fueron: a) los temas publicados por amistades o personas conocidas, los temas que las personas participantes mismas publicaban; b) enumeración de los comentarios alrededor de esos temas; c) evaluación de dichos comentarios; d) argumentos utilizados para sostener, defender o atacar una posición, Además, dentro de la actividad se propuso un juego de roles que permitió a las personas participantes debatir un tema resonado en las redes sociales, en el cual debían interpretar un posicionamiento que no necesariamente era el personal. La guía fue evaluada mediante la realización de una entrevista grupal que sirvió de prueba piloto. Cada entrevista grupal fue dirigida por un miembro del equipo investigador acompañado por otra persona que fungió como observador. Las sesiones tuvieron una duración promedio de una hora y. fueron grabadas en audio y video.

El haber recurrido a la perspectiva de usuarios y usuarias de Facebook fuera del marco de esta red social se consideró un procedimiento válido, puesto que se trata de personas que han crecido con esta y otras redes sociales informatizadas. Por ende, la comunicación que se da dentro y fuera de las redes sociales debe verse como una continuidad más que una separación o una contradicción entre ambos contextos, como dan cuenta estudios acerca de la relación entre auto-concepto y auto presentación en redes sociales y en las relaciones cara a cara (Floridi, 2013; Khan et al., 2016; Ruppel et al., 2016).

\section{Procedimientos de análisis}

Se recurrió un análisis de contenido cualitativo de tipo reductivo a partir de lo propuesto por Mayring (2004). A partir de las preguntas de investigación y la guía de entrevista grupal, se tomó como primer criterio para la definición de categorías los contenidos básicos a ser abordados por la entrevista, a saber: las principales temáticas discutidas en la red y los argumentos que se generan a favor y en contra.

Una vez codificados los grupos de discusión, se procedió a la fase interpretativa, identificando los tipos de temáticas consideradas prototípicas en la discusión en las redes sociales, así como los tipos de razonamiento moral.

La codificación fue llevada a cabo de forma independiente por dos de las investigadoras y en un

Global Media Journal México, 18(35), 95-112, julio - diciembre 2021. 
segundo momento se efectuó un análisis cruzado, para determinar inconsistencias o complementariedades, que permitieran hacer correcciones en la codificación y asegurar la consistencia interna de la codificación.

\section{Resultados}

Un aspecto importante que definió la tematización de las personas participantes fue la coyuntura políticosocial en la que se encontraban inmersas a la hora de las entrevistas grupales. Poco antes de que el equipo investigador iniciara la recolección de datos (abril de 2018), en Costa Rica se habían celebrado las elecciones presidenciales, que requirieron una segunda ronda electoral, ya que ningún partido político obtuvo el porcentaje de votos reglamentado para la presidencia en primera ronda. Particularmente, durante esta segunda contienda, la ciudadanía tuvo que elegir entre un candidato de un partido de corte neopentecostal (Partido Restauración Nacional), que se inclinaba por la defensa de la familia tradicional y otros valores conservadores (Gooren, 2010); y un representante de partido oficialista (Partido Acción Cuidadana), que tenía una agenda política más diversificada, que se basaba en la defensa de los derechos humanos y se mostraba a favor del matrimonio igualitario. Esta polarización política generó un clima de debate activo en la esfera costarricense.

\section{Temas prototípicos}

Se detectaron cuatro temáticas en el centro de las discusiones de las personas entrevistadas, definiendo el contenido de la discusión:

1) Concepciones religiosas: las concepciones religiosas sobre la sexualidad, la identidad sexual y de género, el matrimonio homosexual y la afirmación de los derechos humanos de la población LGBTI y el papel de la institución educativa en la educación sexual de las personas menores de edad.

2) Derechos humanos: la discusión en torno al derecho de las mujeres, la importancia del feminismo en la época actual en contraposición a la violencia de género y el machismo. Los derechos de la población LGBTI y de las personas migrantes

3) Corrupción: la corrupción gubernamental, asociado a enriquecimiento de funcionarios, la participación de altos cargos del gobierno o de los poderes del Estado o la colaboración en el enriquecimiento ilícito de empresas privadas.

4) Xenofobia: se trata del debate en torno a la migración y las amenazas percibidas a la economía o el aumento de la criminalidad percibida.

En la Tabla 1 se aprecian los contenidos básicos que están incorporados en cada uno de los temas. 


\section{Tabla 1}

Contenidos básicos de las temáticas prototípicas en redes sociales reportadas por las y los entrevistados

\begin{tabular}{ll}
\hline Temática general & \multicolumn{1}{c}{ Contenidos básicos (parafraseo) } \\
\hline & -Crítica o defensa del candidato presidencial que \\
& representaba al fundamentalismo religioso en la pasada \\
& elección presidencial \\
& - Religión y fe vs ateísmo \\
& - Estado laico vs Estado confesional \\
& - Educación sexual como tema en la institución escolar en \\
& las escuelas vs educación sexual como asunto \\
& exclusivamente familiar \\
& \\
& - Igualdad de derechos entre hombres y mujeres \\
& - Derechos sexuales y reproductivos de las mujeres \\
& (debate sobre aborto o educación sexual) \\
& - Crítica al feminismo desde posiciones machistas y \\
& conservadoras \\
& - Derechos de la población LGBTI \\
& - Matrimonio homosexual \\
& - Derechos humanos de personas migrantes \\
Derechos humanos & \\
& -Contaminación por parte de la agroindustria piñera y la \\
& tolerancia del gobierno \\
& -Enriquecimiento ilícito y las acciones de diputados o \\
funcionarios de gobierno para favorecer una empresa \\
privada de forma ilegal (caso el Cementazo) \\
- Apropiación de dineros públicos por parte de \\
funcionarios del Estado (referencia general al problema) \\
- Ridiculización de inmigrantes nicaragüenses. \\
- Participación de las y los inmigrantes en la economía \\
- Integración de las personas inmigrantes en la cultura del \\
país de acogida \\
\hline Corrupción & \\
\hline &
\end{tabular}

\section{Argumentación y modelos de razonamiento} moral empleados

En los temas descritos anteriormente se pueden identificar seis tipos de debates o discusiones que refieren a modelos de razonamiento moral. A continuación, se describe cada modelo y se presentan ejemplos de pasajes extraídos de las entrevistas:

\section{Modelo 1}

La evidencia empírica o científica como criterio de legitimización de la información: la argumentación sobre lo discutido gira alrededor de las fuentes en el sentido de que es la información la que sustenta dicha argumentación y si ésta tiene respaldo en hechos comprobados ya sea mediante investigación 
periodística, científica o basada en documentación u observación directa. Los argumentos están sujetos a descalificación si consideran que estos no tienen como fundamento dichas fuentes, sino que se recurre a información inventada, o a supuestos ideológicos o valorativos no respaldables factualmente.

Entran en juego modelos de razonamiento moral de tipo deontológico que, para los participantes en cuestión, implican tomar como norma general la validez del proceder de la ciencia como criterio de verdad. En contraposición entran en conflicto modelos teologistas que anteponen sus creencias religiosas, sobre la base de la apelación a la Biblia como criterio de verdad. Las discusiones que acontecieron en las entrevistas grupales giran en torno a un criterio orientado científicamente o a señalar la ausencia de dicho criterio (ver ejemplos en la Tabla 2).

\section{Tabla 2}

\section{Ejemplos de razonamiento moral deontológico}

1204 M: En el caso de las guías sexuales los que están a favor argumentan como con artículos científicos, con noticias de otros países o con estadísticas de otros países, para demostrar que sí sirve y los que están en contra generalmente se basan en la Biblia y sus creencias para argumentar si están en contra, decir que es que eso está mal, que eso es decirle a las niñas que vayan a, qué sé yo, que vayan a tener relaciones, que queden embarazadas, cuando lo que se busca es justamente lo contrario, digamos los argumentos de ellos es más que todo su creencia y porque me dijeron en la iglesia, por ejemplo.

1405 M: Por ejemplo un día de estos fue el día contra la homofobolesbotransfobia y un señor de Tilarán escribió que él iba a hacer una conferencia en la que él le iba a explicar a gente cómo evitar ser homosexual, eso puso! (risas), y yo me metí y yo veía los comentarios y yo decía qué pasa en el mundo? porque había mucha gente que lo apoyaba y resulta que él al parecer si es una persona estudiada. Porque él sí estudió en la Universidad de Costa Rica, hace muchos años, pero él es odontólogo y él decía que él tenía potestad para hablar de eso, porque él era científico y que él había hecho estudios en eso y que no había un gen homosexual o sea dijo demasiadas cosas y a mí me molestó mucho porque también se estaba defendiendo y dice que no iba a dar como la conferencia solo, sino que lo iba a acompañar otra señora, que además los dos son como demasiado metidos en la iglesia.

\section{Modelo 2}

Los rasgos intrínsecos positivos o negativos atribuidos a individuos o grupos: se refieren a las características individuales que le atribuye el interlocutor a otras personas o grupos, pueden ser referidas a la capacidad intelectual, comunicativa, su ideología política o religiosa. Estas características se asumen como rasgos de personalidad inherentes a las personas, resultando que todo lo que expresen solo puede ser entendido como producto de estas características.

Dentro de estos debates se recurre a modelos de razonamiento identificables con el virtuosismo, que varían dependiendo del contenido de la posición político-ideológica o de la posición dentro de la conflictividad intergrupal. Al describir al endo o al exogrupo, se atribuye virtud en el propio grupo y se 
presenta al exo-grupo como opuesto a las virtudes atribuidas a sí mismos. De esta manera es posible identificar discursos que legitiman el prejuicio contra los emigrantes nicaragüenses a razón de características que se consideran propias de este grupo, como el ser criminales, agresivos o propensos al alcoholismo. Los argumentos acá toman como criterios de virtud la sinceridad, transparencia, o la autenticidad o lo genuino versus la falsedad o la superficialidad (ver ejemplos en la Tabla 3).

\section{Tabla 3}

\section{Ejemplos de razonamiento moral virtualista}

$1907 \mathrm{M}$ : Si usted quiere hacer estallar una noticia, póngale por ejemplo, "nicaragüenses se emborrachan y matan a machetazos a no sé quién". Yo pienso no estamos hablando de que esté mal poner la nacionalidad de la persona sino que cuál es la intención detrás de eso, sabemos que hay mucha xenofobia con respecto a los nicaragüenses, entonces perfectamente se pudo haber puesto, dos hombres matan a machetazos a no sé quién, pero eso no llama la atención.

1204 M: También lo que he visto y es feo, porque yo estoy a favor entonces yo soy el intelectual, yo vine a la universidad...

$1304 \mathrm{M}$ : Y si usted va en contra, es que usted es un conservador, usted es un ignorante, seguro usted no ha estudiado o no sé, entonces la gente dice yo tengo tantas maestrías, yo tengo tantas cosas y argumentaban sacando sus títulos.

1204 M: Sí, esos comentarios a mí me caen mal (risas), porque es como decirle yo tengo razón porque yo sé más que usted, y no porque usted tenga títulos significa que va a saber más que alguien que tal vez no los tenga.

1304 M: Puede ser autodidacta y mucho mejor que uno digamos.

\section{Tabla 4}

\section{Razonamientos morales teologista versus deontologista}

0903 M: Yo he visto un comentario que decía que en realidad las mejoras a los planes deberían de hacerse sin la religión porque la religión ha metido mucha mano en los planes actuales de educación sexual, así que si ahorita estamos mal, culpemos a la religión también, porque la religión ha metido mano.

1103 M: Sí yo vi que cuando Garnier (exminnistro de Educación) presentó los planes de educación sexual, le dijeron que si ya lo había consultado con la comunidad eclesiástica y él... ¿si yo voy a proponer un proyecto de ingeniería lo voy a consultar con un abogado o algo así? o sea ellos no saben entonces ¿para qué?

$0401 \mathrm{H}$ : Ajá y siempre era una posición como de defender algo, porque nos atacan defender la familia tradicional, papá, mamá e hijos y decir que en otros casos sería malo, me acuerdo un comentario que no sé si era broma o qué pero decía, porque si la gente deja de unirse hombre y mujer entonces puede provocar extinción, ya entonces era como eso y como también que no era lo bíblico que Dios había dicho, hombre y mujer, que por eso hombre-hombre no va, ni mujer-mujer, ni cualquier otra variedad, y también decía que los niños necesitaban la figura de la mamá y el papá, pero siempre era como eso familia unida y familia tradicional 


\section{Modelo 3}

Costumbres no religiosas vs costumbres religiosas: Se asume como referencia necesaria y suficiente el punto de vista propio tradiciones, hábitos o costumbres, ya sea asociadas al cristianismo respecto por ejemplo a la diversidad sexual o al modelo de familia cristiano, o, por el contrario, asociados a reconocimiento de matrimonio homosexual, la diversidad de tipos de familia o de las identidades $u$ orientaciones sexuales más allá de la heterosexual.

En estas discusiones se observa la apelación al teologismo y al modelo deontológico con su variante contemporánea, según la cual la racionalidad de la norma está dada por la demostración fáctica, la validez y demostrabilidad científicas (o bien la existencia de normativa internacional producto de la deliberación y el acuerdo racional). Así, por un lado, se apela a las tradiciones religiosas cristianas como criterio para rechazar la educación sexual en la escuela, la homosexualidad o el matrimonio igualitario. Por otro lado, se apela a criterios de la ciencia o de derechos humanos; se parte de la educación y del reconocimiento de los tratados internacionales por parte del país para avalar los temas antes señalados.

Los criterios que toman estas posiciones apelan, por un lado, a la distinción deontologista entre Estado y religión, y por otro lado, a la defensa teologista de los principios religiosos asociados a la familia, como como referencia la noción de Dios como guía última y como norma de acción (ver ejemplos en la Tabla 4).

\section{Modelo 4}

Argumentos basados en la legislación nacional: los argumentos en esta dirección toman como referencia las leyes y normativas nacionales asociadas por ejemplo a matrimonio homosexual, o al concepto de familia. Las posiciones que aquí se esgrimen expresan el modelo contractualista, circunscrito al ámbito nacional; con ello se pretende justificar que la única legislación válida es la nacional. Así se excluye el matrimonio homosexual, al no estar contemplado por la constitución o las leyes locales. La argumentación aquí recurre al criterio contractualista que afirma, en la crítica, la relevancia moral del consenso legítimo y denuncia la supuesta imposición de una institucionalidad jurídica externa (ver ejemplos en la Tabla 5).

\section{Modelo 5}

Derechos humanos como horizonte argumentativo: aquí la argumentación toma como referencia la Declaración Universal de los Derechos Humanos para evaluar matrimonio homosexual, derechos a la identidad, los derechos de las mujeres, y el feminismo, los derechos de las personas migrantes y el derecho a asilo. Estas discusiones toman como modelo de razonamiento un tipo de deontologismo naturalista, al considerar la doctrina internacional sobre los derechos humanos como criterio para definir los derechos de las personas, más allá de la legislación nacional. La argumentación recurre al supuesto valor inmanente de los derechos humanos (ver ejemplos en la Tabla 6 ). 


\section{Tabla 5}

\section{Razonamiento moral contractualista}

3009 H: bueno del matrimonio igualitario la gente discute sobre a quién le corresponde vigilar y quién le corresponde hacer las leyes, entonces la gente anda en una aparente autonomía de qué es el país el que tiene que crear las leyes, que no tenemos que depender de órganos externos (Corte Interamericana de Derechos Humanos) que tengan que decir qué tenemos que hacer, que no tenemos que darles estas decisiones, al final todo el mundo es experto en derecho, que tenemos que darle esas decisiones a la sala constitucional, cuando realmente quien hace las leyes en el país es la asamblea legislativa, porque claramente les conviene tirar eso porque saben que ahí no se va a aprobar algo en lo que ellos están en contra.

\section{Tabla 6}

\section{Razonamiento moral deontologista.}

$1806 \mathrm{M}$ : Bueno yo creo en la igualdad de los humanos entonces si de verdad se considera a las personas migrantes como humanas que tienen derechos: derechos a poder vivir, derechos a poder trabajar, derechos a poder tener acceso a la salud, entonces si consideramos los convenios de los derechos humanos, debemos tomar en cuenta a estas personas, ayudarlas, porque sino estamos no aplicando lo que estamos suscribiendo.

2107 M: nadie le está quitando a usted derechos, lo único que se está peleando aquí es que todas las personas tengan los mismos derechos; no porque se le den derechos a alguien quiere decir que usted va a tener menos (en relación con el matrimonio igualitario).

\section{Modelo 6}

Extremismos: Concepciones de mundo, ideológicas o partidarias que se impone y que descalifican otras existentes. Toman diferentes referentes; puede ser la Biblia o una posición político-ideológica.

Este debate expresa modelos de argumentación teologistas y consecuencialistas; con lo primero, al tomar como criterio de verdad aquello que su religión o libro sagrado dictaminan, y del otro lado, al descalificar la religión a partir del sostenimiento de la laicidad como único criterio. Las discusiones en este tema apelan, entre otros, al reconocimiento del bienestar de todas las personas y a una posición teologista que toma la propia religión como única norma correcta (ver ejemplos en la Tabla 7). 


\section{Tabla 7.}

\section{Razonamientos morales teologista versus consecuencialista}

1103 M: Algo que me llamó a mí la atención de estas elecciones presidenciales fue que varia gente publicó que si usted votó por tal, elimíneme de Facebook, ¡nada que ver! Mucha gente incluso si uno tenía el fondo de la foto de perfil con un marco de alguno de los dos políticos ya lo eliminaban, ¿qué es ese odio tan irracional? Me sorprendió la verdad que se le haya dado tanta importancia, porque tal vez era gente que tenía años de amistad y por votar diferente por las redes sociales se dieron cuenta y ya no se hablan, porque pensaba votar por un determinado candidato ¿usted qué es un hereje o algo así? Entonces como que eso me llamó mucho la atención era como un odio irracional y no fundamentado.

$1304 \mathrm{M}$ : Y digamos me ha tocado mucho este tema porque yo tengo muchos amigos que son gay, entonces la gente argumenta, que ellos se van a ir al infierno, ellos son malos o no pueden adoptar porque después van a violar y yo digo, por Dios, son gente común y corriente, igual que nosotros y por qué hay tanta gente heterosexual que ha violado a sus propios hijos y por qué ellos, porque son homosexuales, ya hay que condenarlos entonces.

$1706 \mathrm{M}$ : por ejemplo, la gente que habla de ideología de género y ni siquiera sabe a qué se refiere, porque es un término que no existe, o que yo opino que las musulmanas no deberían usar el velo, digamos yo no entiendo la religión de ellos y baso mi opinión sólo en prejuicios, no en conocer en realidad en qué consiste la religión de ellos, cómo la viven. Igual acá se critica el Estado laico porque pienso que es tal cosa, no porque realmente investigue qué es para tener fundamento, $o$ veo una noticia ni siquiera la leo y la comento sin fundamento de nada, solo basándome en lo que yo creo que sé o lo que he escuchado.

\section{Discusión}

El presente estudio presenta un aporte al conocimiento de los temas de debate en redes sociales, los argumentos a los que se recurre cuando se discute sobre los temas y los modelos morales que están a la base de dichos argumentos en jóvenes universitarios costarricenses.

Religión, corrupción, derechos humanos y xenofobia son los temas prototípicos de debate en la red social Facebook, según la perspectiva de las personas entrevistadas. Se trata de temas sensibles, que apelan y exigen apelación a razones morales, muchas veces contrapuestas e incluso contradictorias. En una lectura englobante, los temas y las diversas respuestas generadas se vinculan con dos modelos de sociedad en conflicto. Por un lado, una sociedad tradicional que está orientada por la religión cristiana como horizonte ético; por otro lado, una sociedad diferenciada y diversa, que toma el derecho, el multilateralismo, el Estado Laico, y la declaración Universal de Derechos Humanos como horizonte ético. Estas dos formas de sociedad en conflicto se observan en los argumentos y los modelos de razonamiento moral que los sustentan. Estos modelos de sociedad guían la evaluación moral, actuando como heurísticos que se activan cada vez que se tematizan aspectos contenidos en estos modelos de sociedad (Sauer, 2017; Sloman et al., 2009). La forma en que se tematice y la evaluación resultante sería producto de la carga emocional que la acompañe, la cual favorece la evaluación automática y esquemática (Johnen et al., 2018).

Al discutir estos temas entran en juego argumentos como la evidencia empírica o científica que fundamenta o no los argumentos esgrimidos, los rasgos de personalidad o intrínsecos como criterios

Global Media Journal México, 18(35), 95-112, julio - diciembre 2021. 
para explicar el comportamiento de las personas, así como el apelar a costumbres o tradiciones religiosas o no. Junto a ello, se recurre a la legislación nacional o a la Declaración de Derechos Humanos como fundamento de los argumentos para atacar o defender la posición propia. Igualmente, se atribuye dogmatismo como argumento para criticar las posiciones que no se comparten. Cada uno de estos argumentos le permiten dar validez a sus aseveraciones, otorgándole con ello rango de verdad en comparación con las posiciones rivales.

Los principales modelos morales utilizados son el teologismo y el dentologismo, seguido por el virtuosismo y el contractualismo. La recurrencia al teologismo y el deontologismo tiene en común, en este contexto, la apelación a un criterio de inmanencia, por un lado, en las normas aportadas por la religión, y por otro, por las normas aportadas por la Declaración de Derechos Humanos. En ambos casos, la evaluación moral se separa del contexto concreto de interacción, al apelar a estos criterios que lo anteceden. El virtuosismo y el contractualismo, por el contrario, refieren directamente a los actores y las condiciones en las que se produce la norma.

Resulta interesante observar que los participantes recurrieron al consecuencialismo solo de forma complementaria (se debe recordar que el consecuencialismo valora directamente los alcances de las consecuencias de la evaluación moral en la población afectada por las decisiones, y considera fundamental el beneficio de todos sus integrantes). El modelo no resultó prominente para las personas entrevistadas. Esto posiblemente se deba al tipo de temas que estaban presentes en la agenda mediática en el momento de la recolección de información, que centran el debate en los dos tipos de sociedad antes descritos, en los que precisamente predomina criterios de inmanencia para evaluar el devenir de la sociedad. Además, el tipo de comunicación que se da en las redes sociales, asociado a estos temas, parece no apoyar este tipo de modelo, si se quiere más cercano a posturas contextualistas y relativistas $(\sin$ que esto quiera decir que tales modelos lo sean, o que no admitan universalismos).

Sobre este mismo punto es relevante recalcar que la evaluación moral está sujeta a los contextos comunicativos particulares (Mercier \& Sperber, 2017). De allí que la observación hecha anteriormente aplica para los otros contextos evaluativos estudiados, y dependerá no solo de los temas o problemáticas discutidas en las redes sociales, sino también de las y los interlocutores y los contenidos a los que recurren para formar una evaluación moral particular.

Se podría hipotetizar que independientemente del contenido de las evaluaciones realizadas, en el contexto del intercambio de posiciones en Facebook, estas se presentan de forma polarizada, ya que cada una de las diferentes evaluaciones demandan para sí validez. Visto desde el punto de vista del procesamiento cognitivo, a partir de estos sistemas morales, se estructura un heurístico moral altamente esquematizado o rígido, de forma que en contexto del debate en Facebook, más que un intercambio de argumentos lo que se presenta es la afirmación de la propia posición, cada una considerada como la más válida. 
Las particularidades del contenido de la evaluación moral dan cuenta de la señalada polarización entre la defensa de una sociedad tradicional y los grupos que pujan por una secularización y modernización del Estado, la cultura y las formas de vida. El recurso a uno u otro modelo toma como guía los grupos de referencias, que respaldan el fundamento moral de las intervenciones de las personas. Considerado desde un punto de vista socio-cognitivo, estas formas de razonamiento, actúan como heurísticos que se activan en contextos de interacción, específicamente al comentar noticias, comentarios de personas o memes en Facebook y son producto de la respuesta emocional individual como respuesta a los mensajes en las redes sociales (Tarantola, 2017). Esta perspectiva es importante que sea complementada, ateniendo a enfoques socio-lingüísticos y de análisis discursivo, que consideran que las respuestas de las personas usuarias de las redes sociales, están supeditadas a as características de los mensajes presentados por los medios informativos, políticos o líderes de opinión, contribuyendo a la construcción de "otros" y un "nosotros" o de un lector "seguidor" (Mazzuchino 2017), definiendo tanto los términos en que se evalúa la realidad, se debate, pero definiendo también las reglas de juego de la polarización (Gallardo Paúls \& Enguix Oliver, 2016).
En este estudio se recurrió a la perspectiva de personas usuarias de Facebook, recogidas fuera del marco de esta red social. Proceder de esta manera se consideró pertinente, puesto que se trata de usuarios que han crecido con esta y otras redes sociales informatizadas, por lo que entre los contextos comunicativos que se dan dentro y fuera de estas redes sociales lo que acontece es una continuidad más que una separación, o menos aún, una contradicción entre ambos contextos (Floridi, 2013; Khan et al., 2016; Ruppel et al., 2016). No obstante, se considera relevante para investigaciones futuras atender a la estructura comunicativa que se sucede en los intercambios dentro de Facebook, al hacer referencia sobre temas de alta relevancia social.

Así mismo resulta relevante para futuras investigaciones realizar experimentos que permitan estudiar de forma controlada el tipo de temáticas discutidas y su efecto en las evaluaciones y los modelos morales a los que se recurre. Además, se considera relevante analizar el efecto de temáticas que contengan determinadas orientaciones morales predeterminadas en las evaluaciones morales de las y los interlocutores.

\section{Referencias bibliográficas}

Alberici, A. I., \& Milesi, P. (2016). Online discussion, politicized identity, and collective action. Group Processes \& Intergroup Relations, 19(1), 43-59. https://psycnet.apa.org/doi/10.1177/1368430215581430

Brady, W. J., Wills, J. A., Jost, J. T., Tucker, J. A., \& Van Bavel, J. J. (2017). Emotion shapes the diffusion of moralized content in social networks. Proceedings of the National Academy of Sciences, 114(28), 73137318. https://doi.org/10.1073/pnas.1618923114 
Brand, C. (2016). Dual-process theories in moral psychology. Springer.

Cresswell, J. (2013). Qualitative Inquiry and Research Design: Choosing Among Five Approaches. Sage.

Crisp, R. (1998). Routledge philosophy guidebook to Mill on utilitarianism. Routledge.

Flick, U. (2009). An introduction to qualitative research. Sage.

Floridi, L. (2013). The ethics of information. Oxford University Press.

Gallardo Paúls, B., \& Enguix Oliver, S. (2016). Pseudopolítica: el discurso político en las redes sociales. Universidad de Valencia.

Gooren, H. (2010). The Pentecostalization of religion and society in Latin America. Exchange, 39(4), 355-376. https://doi.org/10.1163/157254310X537025

Johnen, M., Jungblut, M., \& Ziegele, M. (2018). The digital outcry: What incites participation behavior in an online firestorm? New Media \& Society, 20(9), 3140-3160. https://doi.org/10.1177\%2F1461444817741883

Jost, J. T., Barberá, P., Bonneau, R., Langer, M., Metzger, M., Nagler, J., Sterling, J., \& Tucker, J. A. (2018). How social media facilitates political protest: information, motivation, and social networks. Political Psychology, 39, 85-118. https://doi.org/10.1111/pops.12478

Kant, E. (1997 [1785]). Groundwork of the Metaphysics of Morals, tr. Mary J. Gregor (1928-1994), with an introduction by Christine Korsgaard (1952-). Cambridge Press.

Karlsen, R., Steen-Johnsen, K., Wollebæk, D., \& Enjolras, B. (2017). Echo chamber and trench warfare dynamics in online debates. European Journal of Communication, 32(3), 257-273. https://doi.org/10.1177\%2F0267323117695734

Khan, S., Gagné, M., Yang, L., \& Shapka, J. (2016). Exploring the relationship between adolescents' self-concept and their offline and online social worlds. Computers in Human Behavior, 55, 940-945. https://doi.org/10.1016/j.chb.2015.09.046

Korsgaard, C. (2000). Las fuentes de la normatividad. UNAM.

Kotras, B. (2018). Le tout plutôt que la partie: Big data et pluralité des mesures de l'opinion sur le web. Revue française de sociologie, 59(3), 451-474. https://doi.org/10.3917/rfs.593.0451

Mazzuchino, M. G. (2017). Los otros y nosotros: configuración discursiva y retórica de los tuits de los presidentes Fernández de Kirchner y Peña Nieto. Tonos digital. Revista de estudios filológicos. (33). https://bit.ly/3yr6ayR

Mayring, P. (2004). Qualitative content analysis. In U. Flick, E. Von Kardoff, \& I. Steinke, A companion qualitative research. (pp.266-269). Sage.

Mercier, H., \& Sperber, D. (2017). The enigma of reason. Harvard University. 
Miller, J. L., Kay, C. S., \& Sanburg, S. (2018). Diamonds in the rough: claims, evidence, and reasoning in Facebook Pollshare.com comments. American Behavioral Scientist, 62(3), 335-352. https://doi.org/10.1177\%2F0002764218769532

Parfit, D. (2011). Reasons and persons. Oxford University Press.

Pérez Sánchez, R. (2016). Adolescencia, socialización y TIC. In PROSIC. Informe Anual hacia la Sociedad de la Información y el Conocimiento 2016. Universidad de Costa Rica: PROSIC. https://bit.ly/3DYJU0p

Quinn, P. L. (2000). Divine command theory. In H. LaFollette (Ed.), The Blackwell guide to ethical theory. Blackwell Publishing.

Quirós, A. (2016). Los adultos y las prácticas de las TIC. In PROSIC. informe Anual Hacia la Sociedad de la Información y el Conocimiento 2016. Universidad de Costa Rica: PROSIC. https://bit.ly/3DYJU0p

Rawls, J. (2005). Political liberalism. Columbia University Press.

Raz, J. (2013). From normativity to responsibility. Oxford University Press.

Ruppel, E. K., Gross, C., Stoll, A., Peck, B. S., Allen, M., \& Kim, S. (2016). Reflecting on connecting: Metaanalysis of differences between computer-mediated and face-to-face self-disclosure. Journal of ComputerMediated Communication, 22(1), 18-34. https://doi.org/10.1111/jcc4.12179

Sauer, H. (2017). Between facts and norms: Ethics and empirical moral psychology. In B. G. Voyer, \& T. Tarantola (Eds.), Moral psychology: A multidisciplinary guide. (pp. 5-28). Springer.

Scanlon, T. (2007). Contractualism and utilitarianism. In R. Shafer-Landau (Ed.), Ethical theory: an anthology. Blackwell Publishing.

Sloman, S. A., Fernbach, P. M., \& Ewing, S. (2009). Causal models: The representational infrastructure for moral judgment. In D. M. Bartels, C. W. Bauman, L. J. Skitka, \& D. L. Medin (Eds.), The psychology of learning and motivation. Vol. 50. Moral judgment and decision making (pp. 1-26). Elsevier.

Slote, M. (2007). Agent-basedd Virtue Ethics. In R. Shafer-Landau (Ed.), Ethical theory: An anthology. Blackwell Publishing.

Tarantola, T. (2017). Cognitive and neural sciences: investigating the moral system. In B. G. Voyer, \& T. Tarantola (Eds.), Moral psychology: A multidisciplinary guide. (pp. 59-79). Springer.

Wright, S., Graham, T., \& Jackson, D. (2016). Third space, social media and everyday political talk. In A. Bruns, G. Enli, E. Skogerbø, A. O. Larsson, \& C. Christensen (Eds.), The Routledge companion to social media and politics. (pp. 74-88). Routledge.

Yoon, C. (2011). Ethical decision-making in the Internet context: Development and test of an initial model based on moral philosophy. Computers in Human Behavior. 27(6), 2401-2409. https://doi.org/10.1016/j.chb.2011.08.007 